\title{
Can Discord Domain-Containing Receptor 2 Mutation Act as a Disease Modifier for PRKAR1A Associated Melanotic Schwannoma?
}

\author{
Erica M. Roman Hernandez ${ }^{a}$ Sri Laxmi Valasareddi ${ }^{b} \quad$ Jarrod Adkison ${ }^{c}$ \\ Henna Awan ${ }^{d}$ Krishnamohan R. Basarakodu ${ }^{b}$ Arash Velayatia \\ a Internal Medicine Residency, Dothan, AL, USA; ${ }^{b}$ Hematology and Oncology Services, \\ Dothan, AL, USA; ${ }^{c}$ Radiation Oncology, Cancer Center, Southeast Health Medical Center, \\ Dothan, AL, USA; ${ }^{d}$ Alabama College of Osteopathic Medicine, Southeast Health, Dothan, \\ $A L$, USA
}

\section{Keywords}

Melanotic schwannoma - Discord domain-containing receptor $2 \cdot$ PRKAR1A

\begin{abstract}
Melanotic Schwannomas are rare neural sheath tumors with distinctive findings of both Schwann cells and melanocytic cells. Recognition of this entity has prompted the importance of distinction from similar tumor types such as melanomas. Early diagnosis facilitates removal of the mass with less risk of local invasion and metastasis. Although previously known as mostly benign lesions, malignant conversion and recurrence are recognized. This paper presents a patient with melanotic schwannoma, describes the distinctive features that will separate it from melanoma, and addresses the possibility of further guided therapy through next-generation sequencing.
\end{abstract}

\section{Introduction}

Melanotic schwannoma was first described arising from a thoracic sympathetic ganglion in 1932 by W. Gilbert Miller. These are rare tumors of neural crest origin arising from common precursors of Schwann cells and melanocytes, accounting for only $1 \%$ of all nerve sheath tumors. Although generally considered benign, about $10 \%$ of reported cases have demonstrated metastatic disease [1]. The underlying premise also recommends long- 
term follow-up due to documented recurrence 5-20 years following resection of the primary tumor $[2,3]$. They can easily be misdiagnosed as melanoma, and often genetic studies or histopathological markers are needed for proper diagnosis and guiding treatment [4]. Due to limited response to treatments such as chemotherapy and radiation, the use of next-generation sequencing in order to guide therapy should be further entertained to assist in creating a patient-directed therapeutic approach and estimating response to treatment.

Here, a case of an aggressive melanotic schwannoma with both PRKAR1A loss of function and Discord Domain-containing Receptor 2 (DDR2) missense mutations is presented in order to emphasize the importance of genome sequencing as a possible guide toward targeted therapy. Up to our knowledge, this is the first case reporting DDR2 mutations in a case of melanotic schwannoma. Finally, this case stresses the underlying malignant potential of melanotic schwannomas by demonstrating both local recurrence and metastasis to solid organs refractory to multiple treatment modalities including surgical excision, radiation, chemotherapy, and targeted therapy.

\section{Case Report}

A previously healthy 31-year-old Caucasian female presented with new-onset right arm numbness, right-sided ptosis, and myosis. CT scan of the chest showed an inhomogeneous enhancing lesion abutting the right superomedial pleural surface, mediastinum, and upper thoracic vertebral bodies measuring $5.4 \times 4.8 \times 5.1 \mathrm{~cm}$. There was mild displacement of the trachea. CT-guided needle biopsy of the mass was suggestive of malignant melanoma (Fig. 1). In IHC staining tumor was positive for S-100, SOX10, MART-1 markers and negative for BRAF, CTNNB1, GNA11, GNAQ, KIT, MAPT2K1, and NRAS (Images not available). PET/CT imaging demonstrated a $5.6 \times 4.8 \mathrm{~cm}$ hypermetabolic right upper thoracic mass (SUV of 14) and no other areas of metabolic uptake. Treatment with ipilimumab and nivolumab for a total of 4 cycles was given.

Repeat PET scan showed no improvement in the disease. Transthoracic mediastinotomy and surgical resection were pursued. The resected tumor appeared well circumscribed with questionable extension of tumor into the vertebral foramina. Pathology confirmed malignant
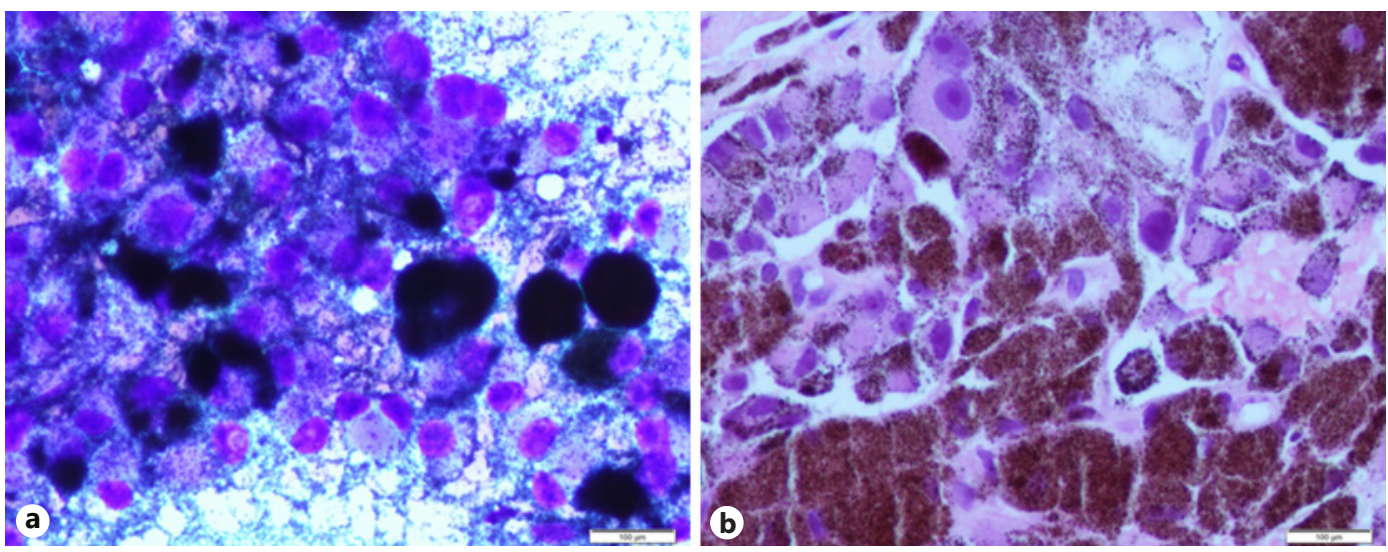

Fig. 1. Biopsy of metastatic lung nodule: melanin-producing cells with nuclear atypia consistent with melanotic schwannoma (HE staining). a Air-dried cytology preparation diff quick stain slide smear of hyperchromatic Schwann cells with heavy melanin pigmentation. b HE slide section of needle core biopsy with hyperchromatic Schwann cells with heavy melanin pigmentation. 
melanotic schwannoma measuring $5.8 \times 4.9 \times 2.5 \mathrm{~cm}$ with positive margins. MRI of the brain showed no evidence of metastatic brain lesions. Presenting symptoms resolved except for mild residual numbness along the medial right arm. Adjuvant radiation therapy was given to the right superior sulcus and paraspinal nerve roots. Serial surveillance imaging showed multiple enlarging nodules at the lung base 17 months after completion of radiation therapy. CT-guided biopsy of lung nodules confirmed recurrent metastatic melanotic schwannoma (Fig. 2).

Next-generation sequencing showed a missense DDR2 mutation of Q231K and loss of function mutation of PRKAR1A (protein kinase, cAMP-dependent, regulatory, type I, alpha). Additional findings included tumor mutational burden -1 Muts $/ \mathrm{Mb}$ and stable microsatellite status. In the absence of standard treatment for this rare tumor, systemic treatment with cytotoxic chemotherapy using an anthracycline versus the tyrosine kinase inhibitor, Dasatinib, to target DDR2 mutation was offered to the patient. Dasatinib was chosen due to a favorable toxicity profile, ease of administration, and low disease burden in this case. Disease progression was noted at 3 months, and patient is currently ongoing second-line treatment with liposomal doxorubicin. Written informed consent was obtained from the patient for publication of this case report and any accompanying images.

\section{Discussion}

Melanotic schwannoma is described as a rare peripheral nerve sheath tumor that arises from a common precursor melanocyte-producing cell. It mostly arises in the spinal nerve roots, with more predominance in the cervical and thoracic spine. However, cases have been reported with solid organ involvement, in locations like the GI tract, soft tissue, and lungs. It is equally prevalent in both males and females, and median age of diagnosis is at 38 years of age [5].

Certain factors that might aid in the diagnosis of melanotic schwannomas can be seen in initial imaging such as calcifications noted in CT imagine and distinctive MRI pattern of hyperintense T1-weighted images followed by variation in T2-weighted imaging from isointense to hypointense $[1,6]$. On gross pathology, melanotic schwannomas would be described as round, hyperpigmented, well-circumscribed masses encapsulated within a thin membrane.
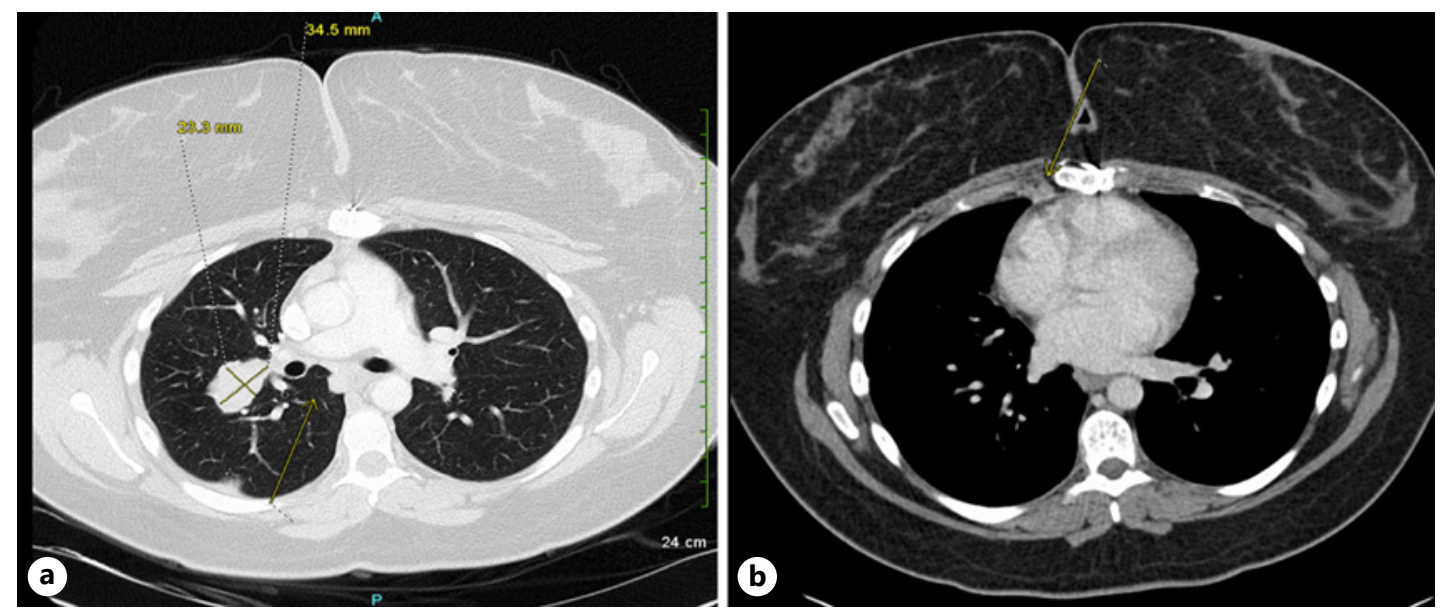

Fig. 2. CT scan showing metastatic recurrence of melanotic schwannoma in the lung (a) and precardiac lymphadenopathy (b). 
Further histologic features used to differentiate melanotic schwannomas from other nerve sheath tumors might include the presence of spindle and epithelioid cells, fascicular arrangement, and melanin-producing cells [7]. Surgical excision is the first-line treatment with radiation therapy reserved only for patients who have incomplete resection of the tumor. The benefit of chemotherapy in these types of tumors has not yet been defined [8]. However, there are chemotherapy regimens used to treat nerve sheath neoplasms that have shown partial response. Examples of such regimens include ifosfamide, doxorubicin, cisplatin, and etoposide as single agent or in combinations [9].

Inactivating germline mutations and deletions in PRKAR1A are associated with Carney Complex, an autosomal dominant syndrome characterized by increased risk of primary pigmented nodular adrenocortical disease, cardiac and other myxomas, endocrine tumors, and schwannomas. The psammomatous type of melanotic schwannoma tends to have an association with Carney Complex. These patients tend to have melanotic schwannomas at a younger age, between their late $20 \mathrm{~s}$ and early $30 \mathrm{~s}$. Somatic mutations in PRKAR1A have also been reported in cardiac myxomas. While loss in expression of the PRKAR1A gene is associated with melanotic schwannoma, these mutations are typically not seen in patients with melanoma [10]. Almost all reported mutations of PRKAR1A that lead to Carney Complex involve substitutions, deletions, insertions, or rearrangements, which do not exceed 15 base pairs. Our patient's loss of function mutation of PRKAR1A without development of Carney Complex could be related to the number of base pairs involved, seeing as a higher involvement leads to more severe variations of the syndrome [11]. There are no target therapies available to address genomic alterations in PRKAR1A.

A missense DDR2 mutation of Q231K was identified in this patient. This mutation is previously reported in the skin squamous carcinoma, and its function is unclear [12]. To our knowledge, DDR2 mutation has not been reported in malignant peripheral nerve sheath tumors. DDR2 is a type I transmembrane receptor tyrosine kinase widely expressed in human tissues, having roles in cell adhesion, migration, proliferation, and survival. There are 2 main types of discoidin (DS) domain receptors, DDR1 and DDR2, which are similar in structure and function. The activation process of these receptors occurs in a slow and continuous fashion. The free collagen-binding DS domain and the collagen-bound DS domain of DDR2 are quite similar [13]. DDR1 has been implicated in different neurological pathologies, including schwannomas [14]. By considering these points, it is possible that the missense nature of the DDR2 mutation present in this patient could have created a structural change mimicking that of DDR1, thus hypotetically, participating in the development of melanotic schwannoma in our patient.

Genomic targeted therapies remain unexplored in this rare tumor type. An example of this is provided by our case report in which DDR2 mutation was identified in our patient leading to initial treatment with Dasatinib. Next-generation sequencing was used to evaluate for actionable targets which identified DDR2 mutation although it was unclear if this was a driver mutation in our patient. DDR2 mutations are present in $1.44 \%$ of all malignant solid tumors, most prevalent in lung adenocarcinoma, colon carcinoma, endometrial endometrioid adenocarcinoma, breast invasive ductal carcinoma, and squamous cell lung cancer. DDR2 mutation promotes tumor proliferation and has been identified in squamous cell lung cancers, prostate cancers, and melanoma [15]. It has also been identified as a driver gene for gastric cancer dissemination and breast cancer metastasis [15].

Dasatinib, one of the most potent kinase inhibitors, has been studied in multiple clinical trials, including lung cancer patients with activating DDR2 mutations. The use of Dasatinib has been shown to reduce tumor growth in squamous lung cancer [16]. However, the highly multi-targeted nature of Dasatinib and its associated toxicity has limited its use [17]. The journey to create therapeutic agents selectively targeted against DDR2 receptors is ongoing.

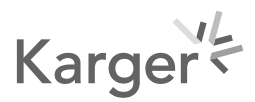


The patient did not have the desired response suggesting DDR2 may not be the driver mutation or possibly not targeted due to the nature of the mutation. The patient's lack of response to Dasatinib treatment is a notable limitation that we encountered. Apart from that, due to the rarity of DDR2 mutation involvement in the development of melanotic schwannomas, we did not have access to a sufficient amount of relevant literature to review. Further research is needed in understanding the possible relationship between DDR2 mutations and melanotic schwannomas.

\section{Conclusion}

Melanotic schwannomas are rare neural sheath tumors that can be easily misdiagnosed and have malignant potential. Late detection of the correct diagnosis can lead to local invasion and poor prognosis. However, with new genetic testing techniques and immunohistochemical studies, such as seen with the case described above, proper identification of these tumors and its driver mutations may be within reach. This might allow for slower progression of the disease and aim toward a more patient-centered treatment. Further research must be done in order to differentiate between benign versus malignant lesions and to determine the efficacy of adjuvant therapy such as radiation and immunotherapy in the treatment of melanotic schwannomas.

\section{Statement of Ethics}

Written informed consent was obtained from the patient for publication of this case report and any accompanying images. Maximum measures were taken to eliminate any patient identifier.

\section{Conflict of Interest Statement}

The authors have no conflicts of interest to declare.

\section{Funding Sources}

The authors did not receive any funding.

\section{Author Contributions}

Eica Roman and Henna Awan were responsible for initial article draft. Sri Valasareddi, Jarrod Adkinson, and Krishna Basarakodu provided care for the patient, revised the paper content, advised on literature attributed, and actively participated in preparation of the paper. Arash Velayati as the lead author, who also organized the efforts in care for the patient, follow-up imaging studies, dividing authors' responsibilities, and critically reviewing the paper.

\section{Karger'/}




\section{Case Reports in Oncology}

\begin{tabular}{l|l}
\hline Case Rep Oncol 2021;14:826-831 \\
\hline DOI: 10.1159/000515331 & $\begin{array}{l}\text { ○ 2021 The Author(s). Published by S. Karger AG, Basel } \\
\text { www.karger.com/cro }\end{array}$ \\
\hline
\end{tabular}

Roman Hernandez et al.: DDR2 Mutations in Melanotic Schwannoma

\section{References}

1 Keskin E, Ekmekci S, Oztekin O, Diniz G. Melanotic schwannomas are rarely seen pigmented tumors with unpredictable prognosis and challenging diagnosis. Case Rep Pathol. 2017;2017:1807879.

2 Collart J, Vandeponseele M, Bosschaert P. Intracranial melanotic schwannomas: rare and distinctive tumors to know due to their risk of recurrence and metastases. J Belg Soc Radiol. 2018 Jan 31;102(1):15.

3 Final diagnosis: malignant psammomatous melanotic schwannoma. Available from https://path.upmc.edu/ cases/case387/dx.htmllast Accessed 2020 Oct 7.

4 Li B, Chen Q. Melanotic schwannoma of thoracic spinal root mimics metastatic melanoma: a potential pitfall for misdiagnosis. Int J Clin Exp Pathol. 2015;8(7):8639-41.

5 Alexiev BA, Chou PM, Jennings LJ. Pathology of melanotic schwannoma. Arch Pathol Lab Med. 2018 Dec; 142(12):1517-23.

6 Khoo M, Pressney I, Hargunani R, Tirabosco R. Melanotic schwannoma: an 11-year case series. Skeletal Radiol. 2016 Jan;45(1):29-34.

7 Topf MC, Pham QH, D’Souza JN, Chaskes M, Tuluc M, Cognetti DM, et al. Pigmented melanotic schwannoma of the neck: report of 2 cases and review of the literature. Ear Nose Throat J. 2019 Feb;98(2):102-6.

8 Faria MH, Dória-Netto RH, Osugue GJ, Queiroz Lde S, Chaddad-Neto FE. Melanotic schwannoma of the cervical spine progressing with pulmonary metastasis: case report. Neurol Med Chir. 2013;53(10):712-6.

9 Steins MB, Serve H, Zühlsdorf M, Senninger N, Semik M, Berdel WE. Carboplatin/etoposide induces remission of metastasised malignant peripheral nerve tumours (malignant schwannoma) refractory to first-line therapy. Oncol Rep. 2002 May-Jun;9(3):627-30.

10 Wang L, Zehir A, Sadowska J, Zhou N, Rosenblum M, Busam K, et al. Consistent copy number changes and recurrent PRKAR1A mutations distinguish Melanotic Schwannomas from Melanomas: SNP-array and next generation sequencing analysis. Genes Chromosomes Cancer. 2015 Aug;54(8):463-71.

11 Horvath A, Bossis I, Giatzakis C, Levine E, Weinberg F, Meoli E, et al. Large deletions of the PRKAR1A gene in Carney complex. Clin Cancer Res. 2008 Jan 15;14(2):388-95.

12 Durinck S, Ho C, Wang NJ, Liao W, Jakkula LR, Collisson EA, et al. Temporal dissection of tumorigenesis in primary cancers. Cancer Discov. 2011 Jul;1(2):137-43.

13 Carafoli F, Hohenester E. Collagen recognition and transmembrane signalling by discoidin domain receptors. Biochim Biophys Acta. 2013;1834(10):2187-94.

14 Vilella E, Gas C, Garcia-Ruiz B, Rivera FJ. Expression of DDR1 in the CNS and in myelinating oligodendrocytes. Biochim Biophys Acta Mol Cell Res. 2019 Nov;1866(11):118483.

15 Henriet E, Sala M, Abou Hammoud A, Tuariihionoa A, Di Martino J, Ros M, et al. Multitasking discoidin domain receptors are involved in several and specific hallmarks of cancer. Cell Adh Migr. 2018;12(4):363-77.

16 Xu C, Buczkowski KA, Zhang Y, Asahina H, Beauchamp EM, Terai H, et al. NSCLC driven by DDR2 mutation is sensitive to Dasatinib and JQ1 combination therapy. Mol Cancer Ther. 2015 Oct;14(10):2382-9.

17 Terai H, Tan L, Beauchamp EM, Hatcher JM, Liu Q, Meyerson M, et al. Characterization of DDR2 inhibitors for the treatment of DDR2 mutated nonsmall cell lung cancer. ACS Chem Biol. 2015 Dec 18;10(12):2687-96. 